\title{
EMPLOYING MINIMIZATION OF GROUP DECISION-MAKING STRESS AND AHP TO OBTAIN RESIDENTS' CONSENSUS
}

\author{
Eizo Kinoshita ${ }^{1}$, Koji Yoshikawa $^{1}$ and Masatake Nakanishi ${ }^{2}$ \\ 'Department of Urban Science, Meijo University \\ 4-3-3 Nijigaoka, Kani,, Gifu, 509-0261 Japan \\ kinoshit@urban.meijo-u.ac.jp/yoshikaw@urban.meijo-u.ac.jp \\ ${ }^{2}$ Faculty of Economics, Nagoya Keizai University \\ 61-1 Uchi-ohkubo, Inuyama, Aichi, 484-8504 Japan \\ nakanishi-m@nagoya-ku.ac.jp
}

\begin{abstract}
In this study, we suggest methods for assigning weightings to residents, by minimization of 'group decision-making stress' and by the AHP method. After formularizing these methods, we consider ways of application to the actual process for obtaining a consensus of residents. That is, we devised role-play experiments supposing selection of a plan from a number of alternatives for an urban improvement project, and confirmed that the results of the proposed methods accorded with those of the experiments.
\end{abstract}

\section{Introduction}

In studies of AHP (Analytic Hierarchy Process), we went beyond the framework of decision-making techniques for multi-purpose plans and examined also the phase of actual consensus-building, targeting particularly application to resident briefings and unofficial meetings. That is, while maintaining fundamental techniques for improving the rationality of selection of alternatives, we focussed on the AHP method as a process model and set out to position the calculating process itself as a system for unifying opinions. The paper considers assimilation of the aims of each resident into the comparison frame of AHP alternatives, methods for presenting the proposed alternative to residents and thus generation of opportunity to unify opinions.

On the basis of these prerequisites, this study supposes a consensus of residents. To achieve a reasonable grading of evaluators (residents, in this study), we propose the method for minimizing group decisionmaking stress and the AHP method. Using the weights calculated by these methods and assigned to participants (evaluators / residents), we use role-play experiments to confirm the appropriateness of presenting specific alternatives as proposed alternatives.

\section{A concept for applying the methods to consensus-forming}

This study also examines methods for rational grading of participants, within an actor-type hierarchical structure. In other words, assuming the importance of appropriate weightings for the evaluators, we suggest application of the group decision-making stress minimization approach, taking into account each evaluator's original preferences to reduce the sum total of each person's frustration. Furthermore, we suggest application of the AHP method for fixing evaluators' weights in advance, by way of calculating each person's influence on other members of the group.

\section{Applying the method for minimizing group decision-making stress towards consensus-forming}

The method for minimizing group decision-making stress suggested in this paper was applied to a practical residents' consensus-forming situation as outlined below. As the pre-process of arranging 
evaluators' grades at appropriate levels, in line with the concept of minimizing the sum value of stress, we conducted comparisons of alternatives by the regular AHP method. Setting uniform weights for all evaluators, we derived the estimated values of alternatives as a group. At this point we calculated the preprocess stress, $S$.

The weight assigned to each evaluator $W^{*}$, calculated after minimizing stress, is used in obtaining the estimated values of alternatives as a group after minimizing stress. On confirming that the sum value of stress has fallen, the alternative with the highest estimated value is presented as the proposed alternative from group decision-making.

Notes) participants $=$ evaluators $=$ residents

\section{An application of the AHP}

Application of the AHP towards consensus-forming was considered as described below. Expressing the influence of one evaluator as perceived by another as a submatrix, the convergent values in a limited stochastic matrix were equated to the level of "voice" of each person in a consensus-forming situation. This is proposed as a rational grading arrangement.

Figure 1 depicts the respective concepts. An "influence network" represents the mutual influence of evaluators, whereas the convergent values of the limited matrix represent the level of influence and appear as a "voice" vector for each evaluator.

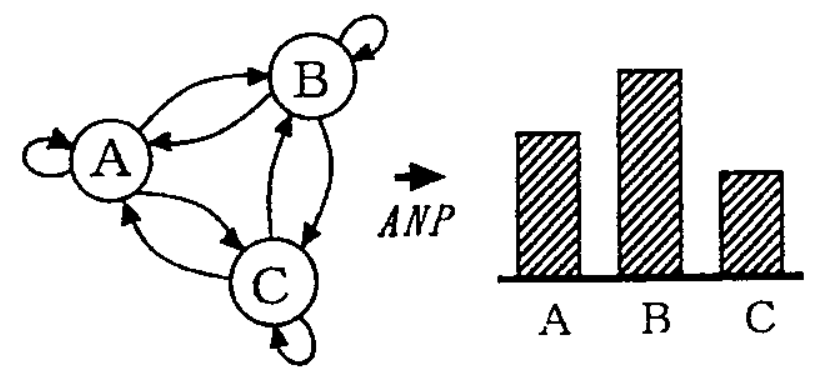

Figure 1. Conceptual diagram of the AHP "voice" vector

The method of calculation is outlined below. During the consensus-forming phase, the residents conducted paired comparisons of "voice" (influence) for all evaluators, including themselves. For Evaluator $k$, this enabled examination of the characteristic "voice" vector $W k$, derived from the comparative matrix. Each person carried out comparisons, and the aggregate data was expressed as the "voice" matrix $W$, shown below as Equation 1.

$$
\mathrm{W}=\left(\mathrm{w}_{1} \mathrm{w}_{2} \ldots \mathrm{w}_{k} \ldots \mathrm{w}_{\mathrm{n}}\right)
$$

If $\mathrm{W}^{n \rightarrow \infty}$, each column vector converges to $w i j^{*}\left(=w^{*}\right)$. It can be assumed that the limit vector of the "voice" matrix corresponds to the final voice in the decision-making phase. That is,

$$
\mathrm{W}^{*}=\left(\mathrm{w}^{*} \mathrm{w}^{*} \ldots \mathrm{w}^{*}\right)
$$

where each element $w i$ of $W^{*}$ can be regarded as the grading given to Evaluator $i$. Using these weightings, we calculated the estimated values of alternatives as a group, presenting the alternative with the highest value as the proposed alternative from decision-making by the group.

\section{Empirical verification of applicability to consensus-forming, by role-play experiments}

\section{Outline of the experiments}

We conducted role-play experiments to confirm the appropriateness of the techniques described in this paper for the resident consensus-forming process. Specifically, the objective of the role-play was to verify 
convergence of opinion and accordance with the theoretical proposed alternative at the conclusion of discussions.

If role-play can confirm theory, depending upon conditions surrounding the decision-making situation and the composition of participants, for each method it should be possible to ensure the theoretical alternative becomes the proposed alternative at the end of discussions, assuming convergence of opinion.

The role-play experiments took place in 1997, on October $27 \& 29$ and November 18, with the cooperation of 27 university students. Discussion focussed on construction of a new road and was held in the form of an unofficial residents' briefing with participation by government officials and others involved in carrying out the project.

Table 1. Role-player composition

\begin{tabular}{c|l|l|l}
\hline & \multicolumn{1}{|c|}{ A: Large City } & \multicolumn{1}{c|}{ B: Medium-sized -City } & C: Agricultural Village \\
\hline \multirow{2}{*}{ Residents } & $\begin{array}{l}\text { Self-employed (male, 52) } \\
\text { Student (female, 20) }\end{array}$ & $\begin{array}{l}\text { Home-maker (female, 35) } \\
\text { Company employee (male, 27) }\end{array}$ & $\begin{array}{l}\text { Farmer (male, 60) } \\
\text { Farmer (male, 38) }\end{array}$ \\
\hline \multirow{2}{*}{ Drivers } & $\begin{array}{l}\text { Driver by occupation } \\
\text { Company employee (male, 35) }\end{array}$ & $\begin{array}{l}\text { Driver by occupation } \\
\text { Home-maker (female, 42) }\end{array}$ & $\begin{array}{l}\text { Driver by occupation } \\
\text { Farmer (male, 70) }\end{array}$ \\
\hline $\begin{array}{c}\text { Project } \\
\text { Organizers }\end{array}$ & $\begin{array}{l}\text { Contractor } \\
\text { Govt. official }\end{array}$ & $\begin{array}{l}\text { Contractor } \\
\text { Govt. official }\end{array}$ & $\begin{array}{l}\text { Contractor } \\
\text { Govt. official }\end{array}$ \\
\hline
\end{tabular}

The roles assigned to the subjects in this series of experiments reflected the composition of an actual residents' briefing, and probable arguments for and against the project. The composition of role-players was organized as set out in Table 2, with careful attention. given to ensuring each subject was clearly apprised of his or her role: To avoid a lack of generality, resulting from set conditions and conditions pertaining to individual subjects, we set up three situations-A: Large City, B: Medium-sized City, C: Agricultural Village. We repeated the experiment three times for each situation, with different subjects (6 each time) role-playing each session. Experimental data was derived from the average values obtained from the experiments. The project alternatives were as follows:

$<$ Alt. $>$ Build no new road (maintain present status)

$<$ Alt.2> Single-lane road without sidewalks

$<$ Alt.3 $>$ Double-lane road with sidewalks

$<$ Alt. $4>$ Four-lane road with sidewalks and median strip

$<$ Alt. $5>$ Elevated road.

\section{Results of the experiments}

Table 2 shows the results derived from the respective experimental sessions. Although the outcome of the 3rd Session differed for the medium-sized city, overall stability of convergence was high, alternation of subjects notwithstanding.

Table 2. Convergence of opinion within the experiments

\begin{tabular}{l|c|c|c}
\hline District & 1st Session & 2nd Session & 3rd Session \\
\hline Large City & Alt.4 & Alt.4 & Alt.4* \\
\hline Medium-sized City & Alt.4 & Alt.4 & Alt.3 \\
\hline Agricultural Village & Alt.3 & Alt.3 & Alt.3 \\
\hline
\end{tabular}

* Opinion converged on the condition that the road would be four-laned. 
Level of accord between experimental results and calculation of alternative weights through minimization of group decision-making stress

Table 3 depicts the results of evaluator weighting calculations. The post-process data refers to evaluator weights obtained through application of the techniques referred to in this paper.

Table 3. Calculated evaluator weights

\begin{tabular}{|c|c|c|c|c|c|c|c|c|}
\hline & $\begin{array}{l}\text { प్ } \\
\text { ర్ }\end{array}$ & \multicolumn{7}{|c|}{ Results of Calculations } \\
\hline \multirow{8}{*}{ 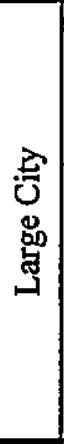 } & \multirow{3}{*}{ 怘 } & Alternative & Alt.1 & Alt.2 & Alt.3 & Alt. 4 & Alt.5 & \\
\hline & & Group estimated value & .193 & .000 & .118 & .355 & .334 & \\
\hline & & Total variance & \multicolumn{6}{|c|}{.00698} \\
\hline & \multirow{5}{*}{$\begin{array}{l}\text { 茄 } \\
\text { \& }\end{array}$} & Alternative & Alt.1 & Alt.2 & Alt.3 & Alt.4 & Alt.5 & \\
\hline & & Group estimated value & .202 & .000 & .131 & .373 & .294 & \\
\hline & & Evaluator & a & b & $\mathrm{C}$ & d & e & f \\
\hline & & Weight & .153 & .201 & .194 & .182 & .124 & .145 \\
\hline & & Total variance & \multicolumn{6}{|c|}{$.00655<$ Stress improvement $=6.1 \%>$} \\
\hline \multirow{8}{*}{ 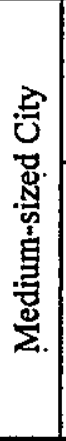 } & \multirow{3}{*}{ Dัّ } & Alternative & Alt.1 & Alt.2 & Alt.3 & Alt.4 & Alt. 5 & \\
\hline & & Group estimated value & .156 & .061 & .285 & .389 & .110 & \\
\hline & & Total variance & \multicolumn{6}{|c|}{.00614} \\
\hline & \multirow{5}{*}{ 㟧 } & Alternative & Alt.1 & Alt.2 & Alt.3 & Alt.4 & Alt.5 & \\
\hline & & Group estimated value & .101 & .041 & .284 & .445 & .129 & \\
\hline & & Evaluator & a & b & c & $\mathrm{d}$ & $\mathbf{e}$ & $\mathbf{f}$ \\
\hline & & Weight & .865 & .177 & .198 & .153 & .198 & .186 \\
\hline & & Total variance & \multicolumn{6}{|c|}{$.00599<$ Stress improvement $=2.4 \%>$} \\
\hline \multirow{8}{*}{ 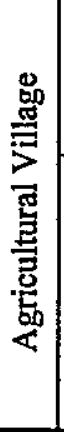 } & \multirow{3}{*}{ 焉 } & Alternative & Alt.1 & Alt.2 & Alt.3 & Alt. 4 & Alt.5 & \\
\hline & & Group estimated value & .378 & .113 & .284 & .182 & .043 & \\
\hline & & Total variance & \multicolumn{6}{|c|}{.00821} \\
\hline & \multirow{5}{*}{$\begin{array}{l}\text { 荡 } \\
\text { 足 }\end{array}$} & Alternative & Alt.1 & Alt.2 & Alt.3 & Alt. 4 & Alt.5 & \\
\hline & & Group estimated value & .377 & .131 & .302 & .159 & .032 & \\
\hline & & Evaluator & $\mathbf{a}$ & b & c & d & e & f \\
\hline & & Weight & .113 & .216 & .125 & .201 & .175 & .171 \\
\hline & & Total variance & & 0740 & ess in & vemer & $9 \%>$ & \\
\hline
\end{tabular}

*The pre-process evaluator weights are all set at a uniform value (=0.167).

*The highest value for any alternative is given in bold type; the 2nd highest is in italics.

In the table above, the figures displayed in bold type correspond to the alternatives with the highest ratings. That is, they are proposed alternatives resulting from group evaluation. These can be rearranged as in Table 4. For the large city, the proposed alternative concurs with the alternative decided on through actual discussion (converged alternative, below). For the medium-sized city, the proposed alternative and the second-largest estimate concur with the two converged alternatives. Meanwhile, the second proposed alternative concurs with the converged alternative in the agricultural village. 
Accordingly, it may be supposed that a certain level of appropriateness as an alternative to 'natural' consensus-forming exists within model scenario valuations of decision-making stress minimization. Although persuasive argument is qualified under the condition of it being a gauge, there is a definite degree of improvement in stress levels, as shown in the tables.

Table 4. Proposed alternatives derived with the technique for minimizing decision-making stress

\begin{tabular}{lcc}
\hline & Largest & 2nd Largest \\
\hline Large City & Alt.4 & Alt.5 \\
\hline Medium-sized City & Alt.4 & Alt.3 \\
\hline Agricultural Village & Alt.1 & Alt.3 \\
\hline
\end{tabular}

Accord between experimental results and alternative weights calculated by the AHP method

A comparative matrix can be derived from the table of the level of influence of each evaluator. The convergent values correspond to the "voice" vectors $w^{*}$, as below.

\begin{tabular}{|c|c|c|c|c|c|}
\hline Evaluator & b & c & d & e & $\mathrm{f}$ \\
\hline $\mathrm{w}_{\mathrm{A}}^{*}=(0.249$ & 0.088 & 0.188 & 0.181 & 0.142 & 0.152 ) \\
\hline$w_{B}^{*}=(0.079$ & 0.243 & 0.102 & 0.227 & 0.128 & $0.106)$ \\
\hline $\mathrm{w}_{\mathrm{c}}{ }^{*}=(0.138$ & 0.174 & 0.092 & 0.207 & 0.156 & $0.118)$ \\
\hline
\end{tabular}

With $w^{*}$ as the weight assigned by 'rational grading', we calculated the overall preference levels for the various alternatives using the normal AHP method.

$\begin{array}{lccccc}\text { Alternative } & \text { Alt.1 } & \text { Alt.2 } & \text { Alt.3 } & \text { Alt.4 } & \text { Alt.5 } \\ \text { Large City } & (0.205 & 0.000 & 0.116 & \mathbf{0 . 3 7 5} & 0.368) \\ \text { Medium-sized City } & (0.116 & 0.000 & \mathbf{0 . 3 5 5} & 0.307 & 0.107) \\ \text { Agricultural Village } & \mathbf{( 0 . 3 8 0} & 0.115 & 0.280 & 0.125 & 0.024)\end{array}$

Here, the figures displayed in bold are the highest preference levels, and correspond to the proposed alternatives. They are expressed in Table 5, in the same format as Table 4.

Table 5. Proposed alternatives derived by the AHP method

\begin{tabular}{lcc}
\hline & Largest & 2nd Largest \\
\hline Large City & Alt.4 & Alt.5 \\
\hline Medium-sized City & Alt.3 & Alt.4 \\
\hline Agricultural Village & Alt.1 & Alt.3 \\
\hline
\end{tabular}

For the large city, the 1st proposed alternatives derived by both the method for minimizing decisionmaking stress and the AHP method concurred with the converged alternatives from actual discussion. For the medium-sized city the 1st and 2nd proposed alternatives concurred with two converged alternatives, 
whereas the 2 nd proposed alternative matched the converged alternative in the agricultural village. Allowing for the limitations, as with the method for minimizing decision-making stress, it may be supposed that a certain level of appropriateness as an alternative to 'natural' consensus-forming exists within model scenario valuations by the AHP method. However, it should be noted that in the case of the large city, the preferred alternative ultimately chosen (Alternative 4) differed significantly from the alternative sought by the evaluator with the largest "voice" (Evaluator $a$ : Alternative 1).

\section{Conclusion}

In this study, we used an evaluator-rating approach to predict the proposed alternative during the group consensus-forming phase. As methods of calculation, we employed minimization of decision-making stress and the AHP technique. We then carried out a series of role-play experiments to confirm the applicability of these methods to the actual consensus-forming stage. As a result, we detected a certain level of applicability. These methods are relatively simple in structure, and appear to embody a high level of mutual assent.

The desired objective is of course a convergence of opinion and a 'natural' formation of consensus. In this study, presentation of the proposed alternatives may be a form of catalyst, in cases where there are low expectations of convergence of opinion. The following examples illustrate.

Creating a "cue/lead" proposal:

Group decision-making usually pressures evaluators towards some form of compromise. The methods outlined above are proposed as "initial cues" towards forming a consensus. If the proposed alternative derived by the above methods is accepted, it will become the converged alternative. If the offered alternatives are unsatisfactory, negotiations can continue until a satisfactory conclusion is reached.

"Unsettling materials":

If matters do not proceed smoothly, a change of perspective may be achieved by using the proposed alternatives and the scenario trial process itself as reference materials. Enlivened debate may thus upset the status quo.

Ultimately, in the practical application, agreement on the methods used for reaching a proposed alternative, publicizing and obtaining agreement on the gradings and other intermediate results may also be effective catalysts in the decision-making process. However, much remains to be done in order to improve methodology and rationality. For example, a prerequisite of the principle of minimizing decision-making stress is normalization of the distribution of opinions. This study did not attempt to verify that point.

Moreover, there is a need for closer approximation of real-life in the technique of verification by role-play experiment. Repeated experiments under varied conditions, with subjects and conditions as close to reality as possible, promise greater reliability of data. 